\title{
EROS study: evaluation between high-dose-rate and low-dose-rate vaginal interventional radiotherapy (brachytherapy) in terms of overall survival and rate of stenosis
}

\author{
Rosa Autorino, MD, PhD!', Luca Tagliaferri, MD, PhD', Maura Campitelli, MD', Daniela Smaniotto, MD',2, \\ Alessia Nardangeli, MD!, Gian Carlo Mattiucci, MD',2, Gabriella Macchia, MD³, Benedetta Gui, MD, Maura Miccò, MD4, \\ Floriana Mascilini, MD5, Gabriella Ferrandina, MD5,6, Gyorgy Kovacs, MD, PhD7 , Vincenzo Valentini, MD 1,2, \\ Maria Antonietta Gambacorta, MD, PhD'.2 \\ IRadioterapia Oncologica, Area Radioterapia Oncologica, Dipartimento Diagnostica per Immagini, Radioterapia Oncologica ed Ematologia, \\ Fondazione Policlinico Universitario A. Gemelli IRCCS, Roma, Italia, ${ }^{2}$ Istituto di Radiologia, Università Cattolica del Sacro Cuore, Roma, Italia, \\ ${ }^{3}$ Radioterapia, Fondazione di Ricerca e Cura Giovanni Paolo II, Università Cattolica del Sacro Cuore, Campobasso, Italy, ${ }^{4}$ Radiologia \\ Diagnostica e Interventistica Generale, Area Diagnostica per Immagini, Dipartimento Diagnostica per Immagini, Radioterapia Oncologica ed \\ Ematologia, Fondazione Policlinico Universitario A. Gemelli IRCCS, Roma, Italia, ${ }^{5}$ Dipartimento Scienze della Salute della Donna \\ e del Bambino, Fondazione Policlinico Universitario A. Gemelli IRCCS, Roma, Italia, ${ }^{1}$ stituto di Clinica Ostetrica e Ginecologica, Università Cattolica \\ del Sacro Cuore, Roma, Italia, ${ }^{7}$ Interdisciplinary Brachytherapy Unit, University of Lübeck - University Hospital S-H, Campus Lübeck, Germany
}

\begin{abstract}
Purpose: To compare the survival and toxicity outcomes in patients with endometrial cancer treated with either highdose-rate (HDR) or low-dose-rate (LDR) vaginal brachytherapy (VBT) following external beam radiotherapy (EBRT).

Material and methods: From January 2000 to December 2014, patients with endometrial cancer after radical hysterectomy with/without pelvic and/or para-aortic lymphadenectomy were treated with adjuvant EBRT (45 Gy, 1.8 Gy/ day to the whole pelvis) and subsequent VBT boost (HDR dose of 7 Gy in one fraction or LDR VBT dose of 25 Gy). The dose was prescribed at $0.5 \mathrm{~cm}$ from the surface of the applicator and the proximal half to two-thirds of the vagina was irradiated. The outcomes of patients were evaluated in terms of local control (LC), overall survival (OS), and rates of adverse events.

Results: We analyzed data of 200 patients treated with EBRT followed by HDR VBT boost in 78 patients and LDR VBT boost in 122 patients. With a median follow-up of 25 months (range, 6-163), 5 -year OS was $98 \%$ and $97 \%$ in the LDR and HDR groups, respectively $(p=0.37)$. The 5 -year LC was similar (93\% in both groups) $(p=0.81)$. In multivariate analyses, none of the factors assessed (age, stage, grade) impacted OS $(p=0.37)$ or LC $(p=0.81)$. Patients treated with LDR VBT after EBRT had higher rates of acute gastrointestinal toxicity. No differences were found in acute genitourinary or hematological toxicities. Late toxicity such as vaginal stenosis was registered during regular follow-up visits and was similar in the two groups $(p=0.67)$.

Conclusions: In our analysis, there were no differences in terms of OS and late toxicity outcomes for patients receiving LDR or HDR VBT. HDR VBT is a safe technique in comparison to LDR VBT.

J Contemp Brachytherapy 2018; 10, 4: 315-320 DOI: https://doi.org/10.5114/jcb.2018.77953

Key words: brachytherapy, endometrial cancer, interventional radiotherapy, vaginal stenosis.

\section{Purpose}

Endometrial cancer is a common gynecological cancer, with approximately 61,000 new cases estimated in 2016. The gold standard for managing the disease is surgery, consisting of a total abdominal hysterectomy and

bilateral salpingo-oophorectomy, with or without lymph node sampling or dissection $[1,2,3,4]$. The use of adjuvant therapy such as external beam radiotherapy (EBRT) and/or vaginal brachytherapy (VBT) is based on disease stage and risk factors for recurrence, and can be individualized.
\end{abstract}

Address for correspondence: Maura Campitelli, MD, Department of Diagnostic Imaging, Radiation Oncology and Hematology, Radiation Oncology Unit, Fondazione Policlinico Universitario A. Gemelli IRCSS, Rome, Italy, Largo A. Gemelli, 00168 Rome, Italy, phone: +39 335 6619855,

๑ e-mail: campitelli.maura@gmail.com 
Historically, low-dose-rate (LDR) brachytherapy has been used to treat endometrial cancer [5]. In the 1970s, high-dose-rate (HDR) brachytherapy was developed to overcome some of the potential disadvantages of LDR brachytherapy such as radiation exposure to the professional staff, need for hospitalization, risk of thromboembolism due to bed immobilization, and patient discomfort.

One potential late side effect of brachytherapy is vaginal stenosis, which occurs from irreversible mucosal damage from radiation. Severe stenosis may lead to the inability to perform an adequate pelvic examination and to sexual dysfunction, and can negatively affect the patient's quality of life [6,7].

Few studies compare LDR and HDR VBT in the treatment of endometrial cancer, especially in terms of late toxicity. We retrospectively analyzed our institutional results for endometrial cancer patients treated with either HDR or LDR VBT after EBRT in terms of survival and safety.

\section{Material and methods}

\section{Patient selection}

Patients diagnosed with endometrial cancer who underwent EBRT and VBT from January 2000 to December 2014 were retrospectively considered for this study. All data were selected from the intranet hospital multidivisional electronic database, Spider's Net [8] in the frame of the COBRA system [9]. Inclusion criteria were histologically proven diagnosis of endometrial cancer, EBRT $>40 \mathrm{~Gy}$, VBT boost, and follow-up > 6 months. In all cases, the indication for the treatment was discussed in a multidisciplinary gynecologic tumor board. All patients provided informed consent.

\section{Patient treatment}

All patients were treated at our institution with total hysterectomy and bilateral salpingo-oophorectomy, with or without lymph node dissection. All patients received EBRT to the pelvic lymph nodes at a dose of $45 \mathrm{~Gy}$, $1.8 \mathrm{~Gy} /$ day, for 5 days/week, performed with a 3-dimensional technique. A sub-sequential VBT boost was administered in all patients in one fraction. Depending on the institutional protocol in use at the time of therapy, one of two modalities were used: VBT boost in LDR, with a total dose of $25 \mathrm{~Gy}$, or VBT boost in HDR, with a dose of 7 Gy in one fraction prescribed at $0.5 \mathrm{~cm}$ from the surface of the applicator. The proximal half to two-thirds (based on clinical presentation) of the vagina was irradiated. Vaginal cylinders ranged from $2.0-3.5 \mathrm{~cm}$.

\section{External beam radiotherapy technique}

For EBRT, simulation and treatment were performed with the patient in a prone position using an up-down table device [10] aimed to reduce the small bowel volume in the treatment field. A computed tomography (CT) scan was used for EBRT planning in all patients. The pelvic volume (clinical target volume [CTV] 2) was defined as the upper two-thirds of the vagina (if not involved) or the whole vagina (if involved at pathologic evaluation), obturator lymph nodes, external iliac nodes, internal iliac nodes, and presacral nodes. Because of organ motion and setup uncertainties, a planning target volume (PTV) 2 was defined by adding an $8 \mathrm{~mm}$ isotropic margin to the CTV.

\section{Brachytherapy technique}

Before 2012, patients who received brachytherapy were treated using the LDR system, which delivers a highdose of radiation at a low-dose-rate from an applicator placed temporary in the organ. The patients had bowel preparation and were on a constipating regimen, and prophylactic measures were taken for thromboembolic risk.

After disinfecting the external genitalia and positioning the urinary catheter and rectal probe, vaginal applicator of varying circumferences was inserted without anesthesia or under local anesthetic.

Antero-posterior and lateral radiographs or CT-simulation after 2010 were performed to identify the target and organs at risk (bladder and rectum). The LDR VBT dose was $25 \mathrm{~Gy}$, which was prescribed at $0.5 \mathrm{~cm}$ from the surface of the applicator. The proximal half to two-thirds of the vagina was irradiated. Vaginal cylinders ranged from $2.0-3.5 \mathrm{~cm}$. The treatment plan was performed using PLATO, or OncentraBrachy after 2010 with a 3-dimensional technique. The treatment was performed by the Selectron device with ${ }^{137} \mathrm{Cs}$ before 2010, and with the MicroSelectron device with an HDR ${ }^{192}$ Ir source after 2010.

For HDR treatments from 2012, the same preparation as for LDR treatment was required and a similar vaginal applicator insertion was performed. The patient was simulated using a CT scan and the treatment plan was performed on the OncentraBrachy treatment planning system (TPS) with a 3-dimensional technique. The HDR VBT dose prescribed was $7 \mathrm{~Gy}$ in one fraction at $5 \mathrm{~mm}$ from the applicator surface by a MicroSelectron device with an ${ }^{192}$ Ir source. The proximal half to two-thirds of the vagina was irradiated. Vaginal cylinders ranged from $2.0-3.5 \mathrm{~cm}$.

\section{Patient follow-up}

After therapy, all patients were periodically evaluated every 3 months during the first year, every 4 months during the second year, every 6 months until 5 years, and annually after 5 years. According to an internal QA protocol, gastrointestinal and genitourinary adverse events were evaluated according to the RTOG (Radiation Therapy Oncology Group) morbidity grading system scale (Table 1) [11], based on information reported in the clinical chart, while vaginal mucosal toxicity was defined according to the CTCAE version 4.0. It was assessed from the symptoms reported by the patient, and any mucosal changes noted on speculum and bimanual exams at each visit.

\section{Statistical analyses}

Statistical analysis was performed using MedCalc software (www.medcalc.be). Overall survival (OS) was calculated using the Kaplan-Meier method. Univariate and multivariate logistic regressions (T-test) for toxicity analyses were performed. 
Table 1. RTOG late radiation morbidity scoring schema

\begin{tabular}{|c|c|c|c|c|c|}
\hline Organ/tissue & Grade 0 & Grade 1 & Grade 2 & Grade 3 & Grade 4 \\
\hline $\begin{array}{l}\text { Small/large } \\
\text { intestine }\end{array}$ & None & $\begin{array}{l}\text { Mild diarrhea; } \\
\text { mild cramping; } \\
\text { bowel movement } \\
5 \text { times daily; } \\
\text { slight rectal } \\
\text { discharge or bleeding }\end{array}$ & $\begin{array}{l}\text { Moderate diarrhea } \\
\text { and colic; bowel } \\
\text { movement }>5 \text { times } \\
\text { daily; excessive } \\
\text { rectal mucus or inter- } \\
\text { mittent bleeding }\end{array}$ & $\begin{array}{l}\text { Obstruction or } \\
\text { bleeding, } \\
\text { requiring surgery }\end{array}$ & $\begin{array}{l}\text { Necrosis/perforation } \\
\text { fistula }\end{array}$ \\
\hline Bladder & None & $\begin{array}{c}\text { Slight epithelial } \\
\text { atrophy; minor telan- } \\
\text { giectasia (microscopic } \\
\text { hematuria) }\end{array}$ & $\begin{array}{l}\text { Moderate frequency; } \\
\text { generalized telan- } \\
\text { giectasia; intermit- } \\
\text { tent macroscopic } \\
\text { hematuria }\end{array}$ & $\begin{array}{l}\text { Severe frequency } \\
\text { and dysuria; } \\
\text { severe generalized } \\
\text { telangiectasia (often } \\
\text { with petechiae); } \\
\text { frequent hematuria; } \\
\text { reduction in bladder } \\
\text { capacity (<150 cc) }\end{array}$ & $\begin{array}{l}\text { Necrosis/contracted } \\
\text { bladder (capacity } \\
<100 \text { cc); severe } \\
\text { hemorrhagic cystitis }\end{array}$ \\
\hline
\end{tabular}

Table 2. Patient characteristics

\begin{tabular}{|c|c|c|c|}
\hline Patient characteristics & Total & HDR & LDR \\
\hline Age (median) & $63(31-88)$ & $64(36-88)$ & $62(47-88)$ \\
\hline \multicolumn{4}{|l|}{ Histology } \\
\hline Adenocarcinoma & $200(100 \%)$ & $78(39 \%)$ & $122(61 \%)$ \\
\hline \multicolumn{4}{|l|}{ Stage } \\
\hline IA & $14(7 \%)$ & $12(85 \%)$ & $2(15 \%)$ \\
\hline $\mathrm{IB}$ & $55(28 \%)$ & $39(70 \%)$ & $16(30 \%)$ \\
\hline IC & $59(29 \%)$ & $2(3 \%)$ & $57(97 \%)$ \\
\hline$\|$ & $44(22 \%)$ & $10(22 \%)$ & $34(78 \%)$ \\
\hline IIIA & $13(6.5 \%)$ & $5(38 \%)$ & $8(62 \%)$ \\
\hline$\| I I B$ & $3(1.5 \%)$ & $2(66 \%)$ & $1(34 \%)$ \\
\hline IIIC & $12(6 \%)$ & $8(66 \%)$ & $4(34 \%)$ \\
\hline \multicolumn{4}{|l|}{ Grading } \\
\hline Well differentiated & $25(12.5 \%)$ & $4(16 \%)$ & $16(84 \%)$ \\
\hline Moderately well-differentiated & $97(48.5 \%)$ & $32(33 \%)$ & $66(67 \%)$ \\
\hline Poorly differentiated & $78(39 \%)$ & $42(54 \%)$ & $40(46 \%)$ \\
\hline \multicolumn{4}{|l|}{ Miometrial invasion } \\
\hline$<50 \%$ & $55(27.5 \%)$ & $24(44 \%)$ & $30(66 \%)$ \\
\hline$>50 \%$ & $145(72.5 \%)$ & $54(37 \%)$ & $92(63 \%)$ \\
\hline
\end{tabular}

$L D R$ - low-dose-rate, $H D R$ - high-dose-rate

\section{Results}

We analyzed a total of 200 patients treated with EBRT, which was combined with HDR VBT boost in 78 patients and LDR VBT boost in 122 patients. Patient characteristics are summarized in Table 2.

With a median follow-up of 25 months (range, 6-163 months), the 5-year OS was $98 \%$ vs. $97 \%$ in the LDR and HDR group, respectively $(p=0.37$ ) (Figure 1$)$. The 5 -year local control rate (LC) was $93 \%$ in both groups $(p=0.81)$ (Figure 2). In multivariate analyses, none of the investigat- ed factors (age, stage, grading, treatment) showed an impact on OS $(p=0.37)$ and LC $(p=0.81)$.

\section{Toxicity}

The treatment was generally well tolerated. The normal tissues at risk for developing acute radiation-related complications after treatment for endometrial cancer are in the genitourinary or gastrointestinal tracts. Grade 3 acute gastrointestinal toxicity (proctitis or diarrhea) were observed in 3 patients in the LDR group and 1 patient in the 


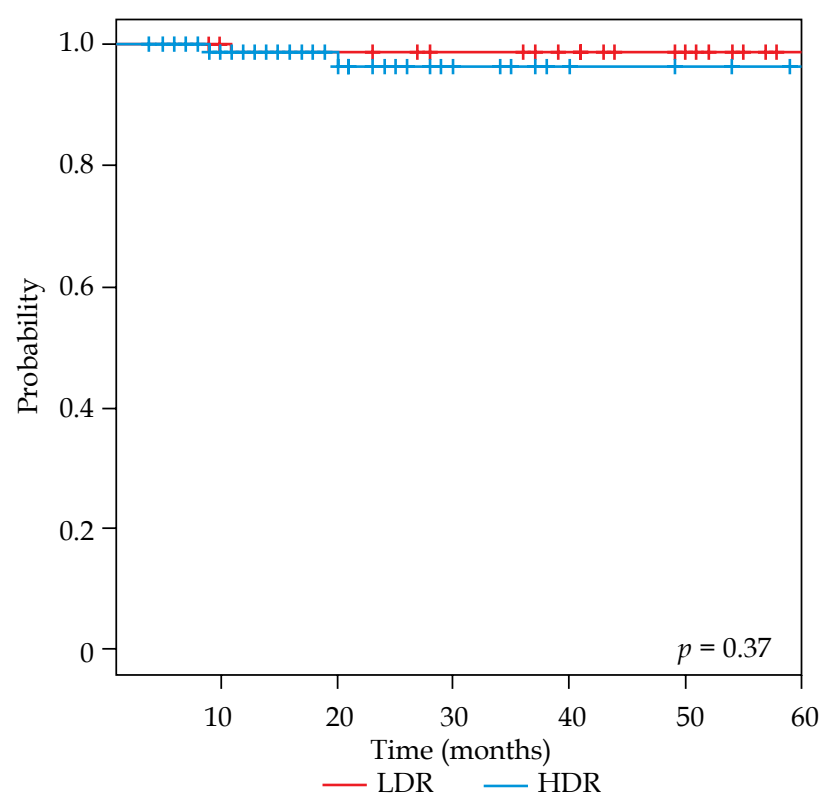

Fig. 1. Overall survival

HDR group $(p=0.0014)$. Genitourinary acute toxicity (severe frequency and dysuria) was greater in the LDR group compared with the HDR group $(p=0.0195)$ (Figure 3).

Regarding vaginal stenosis (reported at least six months from the date of brachytherapy), no significant difference was observed between the two groups $(p=0.67)$; even if comparing the grade $0-2$ vs. grade 3 toxicities between groups, we observed a significant difference $(p=0.004)$ (Figure 4). However, in some patient files, a stenosis was noted but not specified according to the grading scale. Therefore, these data are not available for all patients (16 patients of HDR group and 9 patients for LDR group).

\section{Discussion}

VBT plays an important role in adjuvant treatment of endometrial cancer. The PORTEC 2 trial [12] showed the efficacy of post-operative VBT in improving local control after abdominal hysterectomy/bilateral salpingo-oophorectomy compared with pelvic EBRT. One significant late toxicity described with this treatment is vaginal stenosis. It is due to the combination of adhesions and circumferential fibrosis in the upper vaginal tissue, with consequent narrowing and shortening of the vaginal vault. It can interfere with physical examinations and sexual functioning, and impact patients' quality of life.

In the PORTEC 2 trial, the rate of grade 2 vaginal mucosal atrophy was significantly higher after VBT than after EBRT; in the same study, grade 3 atrophy was reported in $<1 \%$ of the patients receiving EBRT and 2\% of those receiving VBT, despite similar sexual activity rates. However, specific rates of vaginal stenosis were not reported. Also, in the recently published study on the longterm health-related quality of life from the PORTEC-2 trial reported by de Bouer [13], vaginal stenosis was not considered.

Furthermore, in the literature, there are only a few studies comparing HDR and LDR VBT in the treatment of

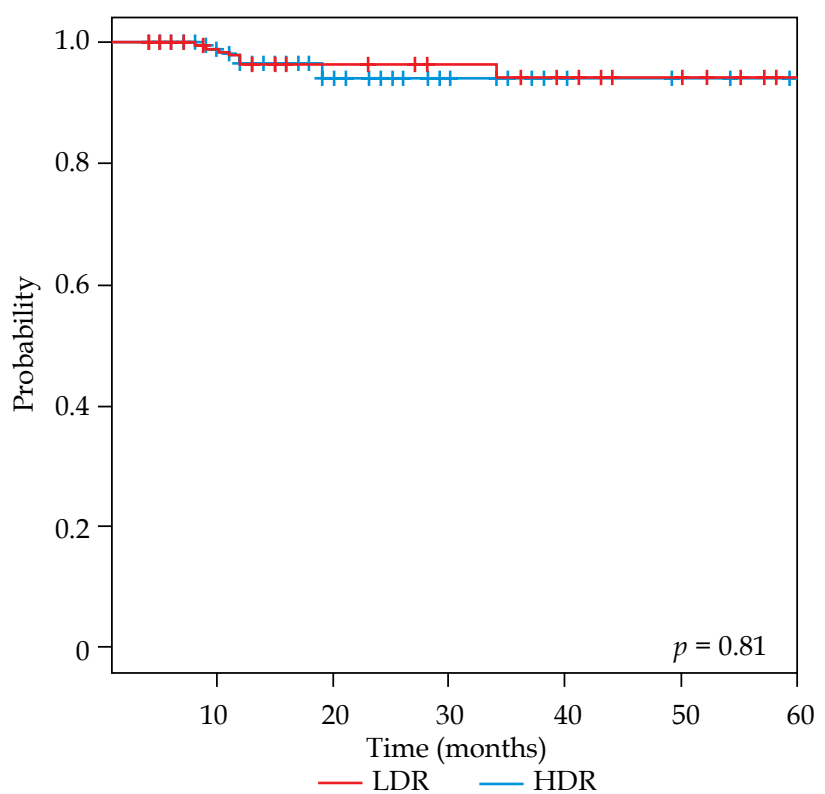

Fig. 2. Local control

endometrial cancer. Bekerus et al. [14] published a study with similar survival results for the LDR and HDR groups but higher rates of late complications in the LDR group. In the same year, Rauthe et al. [15] showed that HDR after-loading irradiation improved the survival rate, with more frequent fistula formation in the HDR group, which was mainly produced by the low number of fractions and high single doses. In another retrospective study performed by Fayed et al. [16], intravaginal HDR VBT treatment results were compared to LDR VBT results, and no differences in survival and toxicity outcomes were observed. Neither of these studies reported on vaginal stenosis.

The observations from our study suggest that 5-year overall survival and 5-year local control were similar in the two groups. Subgroup analysis stratified by age, stage, grade, and treatment did not show statistically significant differences in terms of OS and LC. The occurrence of a higher rate of acute gastrointestinal toxicities in the LDR VBT group was probably caused by the development of the radiation technique over the years of this study. Regarding the technique used for brachytherapy, a 3-dimensional modality was introduced in 2010. No differences in vaginal stenosis rates were found between the two different VBT groups.

Limitations of this study are the same as those of any retrospective single-institutional analysis. Diagnostic imaging, surgical technique, and radiotherapy technique have changed over the years. However, the main limitation of this study is that we compared two different schedules of adjuvant treatments with different total doses in terms of EQD2: 7 Gy in HDR brachytherapy is equivalent to $9.92 \mathrm{~Gy} \mathrm{EQD}_{2}$ with an a/b of 10 and $14 \mathrm{~Gy}$ $\mathrm{EQD}_{2}$ with an $\mathrm{a} / \mathrm{b}$ of 3 , while 25 Gy in LDR are equivalent to 25 Gy with $a / b$ of 3 . The HDR schedule was as suggested by ABS guidelines, while the LDR schedule was defined according to the institutional guidelines, and the delivered dose was probably higher than that of other studies reported in literature. 

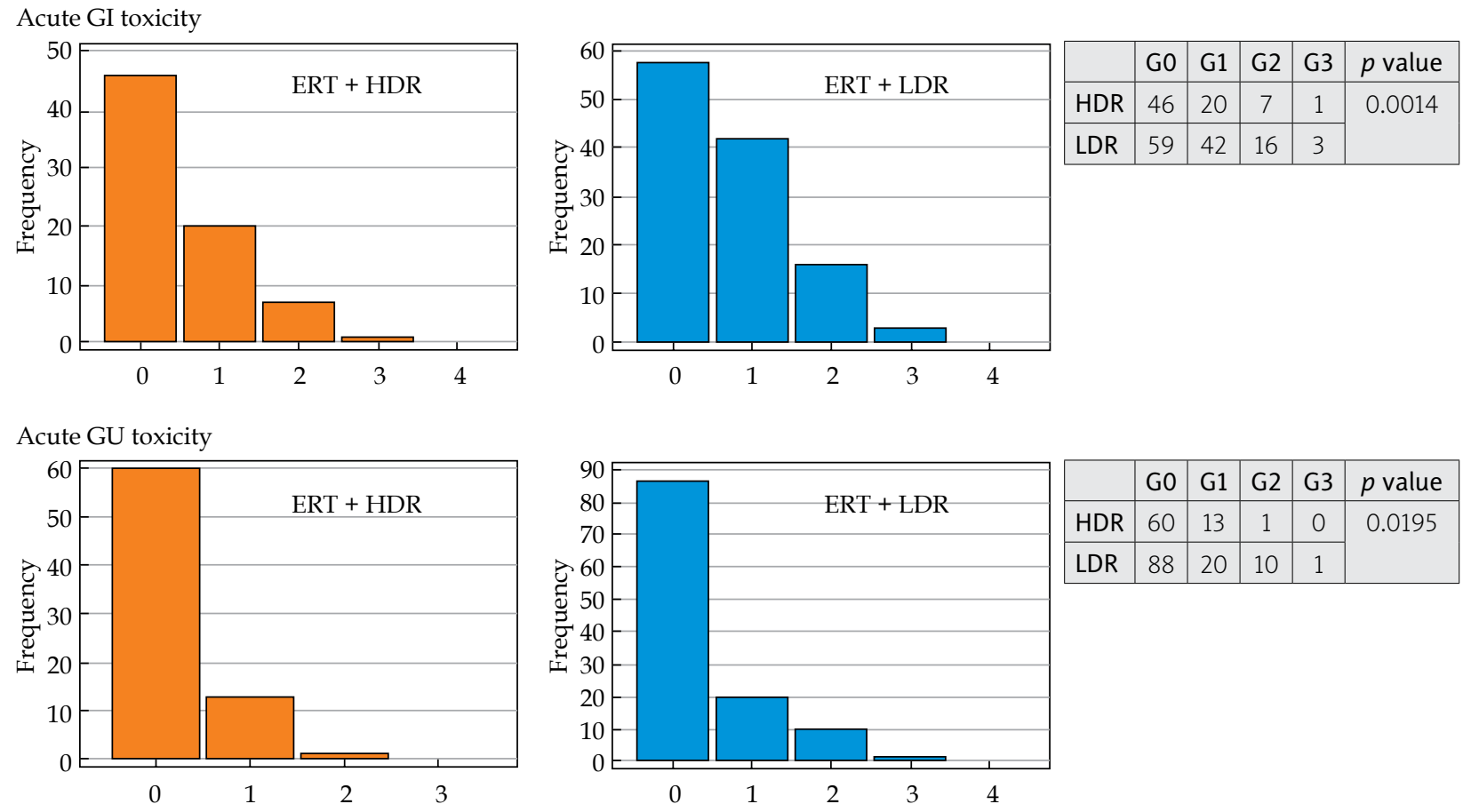

Acute hematological toxicity
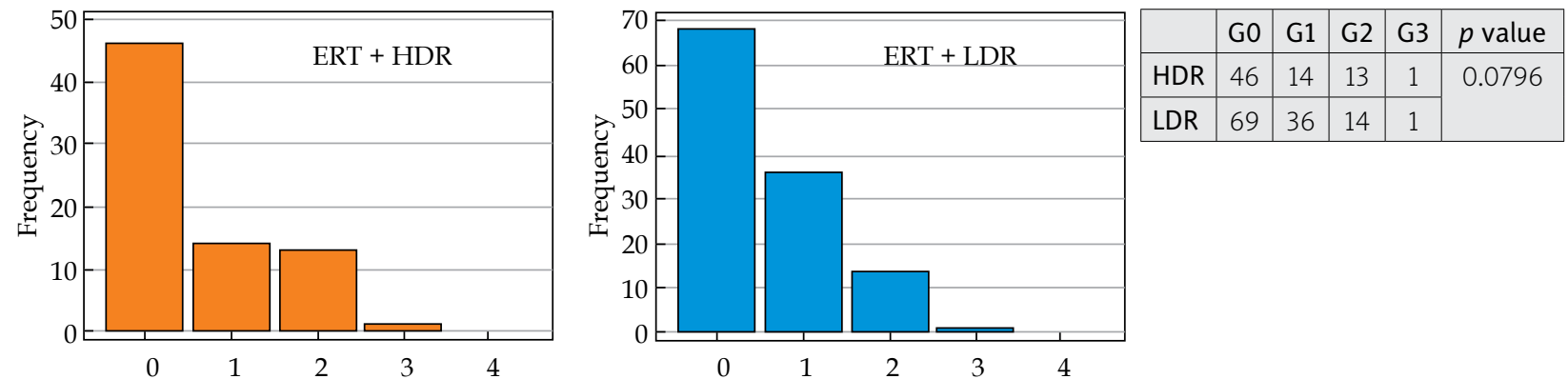

Fig. 3. Acute treatment complications correlated with treatment
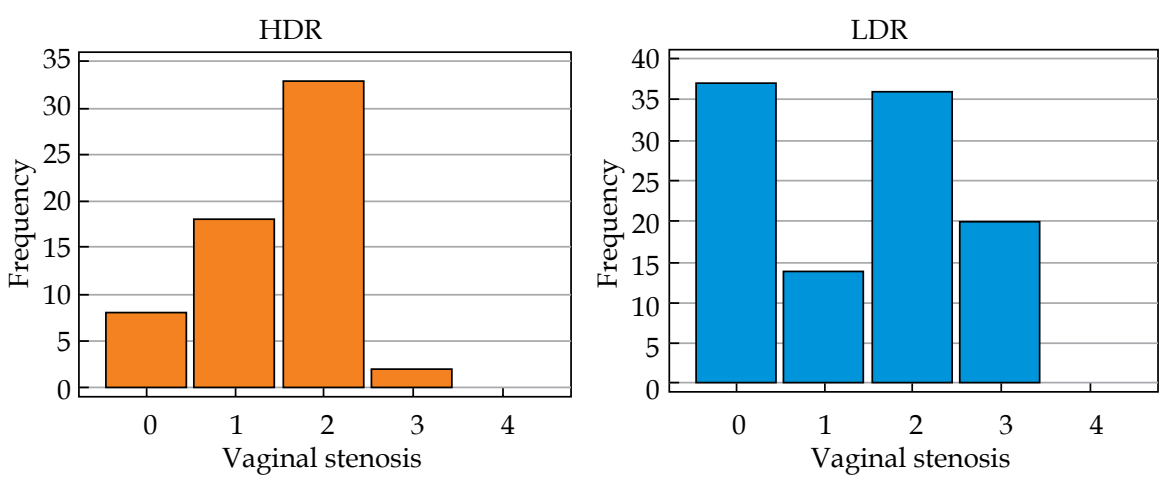

\begin{tabular}{|l|c|c|c|c|c|c|}
\hline & G0 & G1 & G2 & G3 & NA & $p$ value \\
\hline HDR & $8(10 \%)$ & $18(23 \%)$ & $33(42 \%)$ & $3(4 \%)$ & $16(21 \%)$ & 0.67 \\
\hline LDR & $37(30 \%)$ & $18(14 \%)$ & $36(29 \%)$ & $22(18 \%)$ & $9(9 \%)$ & \\
\hline & G0-2 & G3 & NA & p value \\
\hline HDR & $59(75 \%)$ & $3(4 \%)$ & $16(21 \%)$ & \multirow{2}{*}{0.004} \\
\hline LDR & $91(75 \%)$ & $22(18 \%)$ & $9(7 \%)$ & \\
\hline
\end{tabular}

Fig. 4. Vaginal stenosis correlated with treatment 
Another limitation of this retrospective study is that in some patient files the stenosis was described but not defined according the grading scale. These data are not available for all patients; for this reason, in the tables and graphs only the patients with available grade evaluation are reported.

The inter-observer uncertainty does not play a role in this analysis, since the same specialist recorded all toxicity data over the entire period of the present study.

Our interpretation of the data suggests less vaginal stenosis and acute gastrointestinal and genitourinary toxicity with HDR VBT than LDR VBT. However, it must be emphasized that the equivalent dose with HDR VBT was lower than that with LDR VBT. Probably there was a reduction in toxicity from using a lower overall dose. Nevertheless, this is still intriguing because lowering the dose did not result in a difference in local control.

\section{Conclusions}

This retrospective study did not show differences in late toxicity outcomes and in local control values for patients receiving 25 Gy LDR or 7 Gy HDR VBT. With the introduction of HDR VBT, we observed a reduction in gastrointestinal and genitourinary toxicities without worsening of vaginal stenosis rates compared to LDR VBT-related data. In post-operative endometrial cancer, 7 Gy HDR VBT can be considered as safe, efficient, and complementary to $45 \mathrm{~Gy}$ conventional fractionated EBRT.

\section{Disclosure}

The authors report no conflict of interest.

\section{References}

1. American Cancer Society (US). What are the key statistics about endometrial cancer? [Internet]. Atlanta (GA): American Cancer Society, Inc. Available from http://www.cancer. $\mathrm{org} /$ cancer/endometrialcancer/detailedguide/endometrial - Uterine-Cancer-Key-Statistics; 2014 (updated 2014 Feb 3; cited 2014 Aug 23).

2. Creasman WT, Morrow CP, Bundy BN et al. Surgical pathologic spread patterns of endometrial cancer. A Gynecologic Oncology Group Study. Cancer 1987; 60: 2035-2041.

3. Creutzberg CL, van Putten WL, Koper PC et al. Surgery and postoperative radiotherapy versus surgery alone for patients with stage-1 endometrial carcinoma: multicentre randomised trial. Post-operative radiation therapy in endometrial carcinoma. Lancet 2000; 355: 1404-1411.

4. Keys HM, Roberts JA, Brunetto VL et al. A phase III trial of surgery with or without adjunctive external pelvic radiation therapy in intermediate risk endometrial adenocarcinoma: A Gynecologic Oncology Group study. Gynecol Oncol 2004; 92: 744-751.

5. Draghini L, Maranzano L, Casale M et al. Definitive threedimensional high-dose-rate brachytherapy for inoperable endometrial cancer. J Contemp Brachytherapy 2017; 9: 118-123.

6. Podczaski E, Kaminski P, Gurski K et al. Detection and patterns of treatment failure in 300 consecutive cases of "early" endometrial cancer after primary surgery. Gynecol Oncol 1992; 47: 323-327.

7. Bergmark K, Avall-Lundqvist E, Dickman PW et al. Patientrating of distressful symptoms after treatment for early cervical cancer. Acta Ostetr Gynecol Scand 2002; 81: 443-450.
8. Valentini V, Maurizi F, Tagliaferri L et al. Spider: managing clinical data of cancer patients treated through a multidisciplinary approach by a palm-based system. Ital J Public Health JPH 2008; 6.

9. Tagliaferri L, Kovács G, Autorino R et al. ENT COBRA (Consortium for Brachytherapy Data Analysis): interdisciplinary standardized data collection system for head and neck patients treated with interventional radiotherapy (brachytherapy). J Contemp Brachytherapy 2016; 8: 336-343.

10. Capirci C, Polico C, Mandoliti G. Dislocation of small bowel volume within box pelvic treatment fields, using new "up down table" device. Int J Radiat Oncol Biol Phys 2001; 51: 465-473.

11. https://www.rtog.org/ResearchAssociates/AdverseEventReporting/RTOGEORTCLateRadiationMorbidityScoringSchema.aspx

12. Nout RA, Putter H, Jürgenliemk-Schulz IM et al. Five-year quality of life of endometrial cancer patients treated in the randomised Postoperative Radiation Therapy in Endometrial Cancer (PORTEC-2) trial and comparison with norm data. Eur J Cancer 2012; 48: 1638-1648.

13. De Boer SM, Nout RA, Jürgenliemk-Schulz IM et al. LongTerm Impact of Endometrial Cancer Diagnosis and Treatment on Health-Related Quality of Life and Cancer Survivorship: Results from the Randomized PORTEC-2 Trial. Int J Radiat Oncol Biol Phys 2015; 93: 797-809.

14. Bekerus M, Durbaba M, Frim O et al. Comparison of HDR and LDR results in endometrium cancer. Sonderb Strahlenther Onkol 1988; 82: 222-227.

15. Rauthe G, Vahrson H, Giers G. Five-year results and complications in endometrium cancer: HDR afterloading vs. conventional radium therapy. Sonderb Strahlenther Onkol 1988; 82: 240-245.

16. Fayed A, Mutch DG, Rader JS et al. Comparison of highdose-rate and low-dose-rate brachytherapy in the treatment of endometrial carcinoma. Int J Radiat Oncol Biol Phys 2007; 67: $480-484$ 